\title{
A Problem of Capacity, but Whose? The Hospitalists' Discharge Dilemma and Social Determinants of Health
}

\author{
Hilary J. Mosher, MFA, MD ${ }^{1,2 *}$, Laurel J. Lyckholm, MD²
}

${ }^{1}$ Center for Comprehensive Access and Delivery Research and Evaluation (CADRE), lowa City VA Healthcare System, lowa City, lowa; ${ }^{2}$ Department of Internal Medicine, University of lowa Carver College of Medicine, lowa City, lowa.

For a number of years, those challenged with improving discharge transitions and preventing readmissions have suggested more-more case managers, more checklists and systems, more discharge pharmacists; and better-better communication, better medication reconciliation, better discharge documentation, better follow-up. In a study by Chan Carusone et al., ${ }^{1}$ high-need, high-complexity patients receiving treatment at Casey House, a specialized urban hospital providing inpatient and community programs, were afforded a full complement of discharge planning and posthospitalization services. Despite these services, the patients achieved little success in maintaining their health and following their discharge plans after hospitalization.

This longitudinal qualitative study detailing the lived experience of discharge extends our knowledge of challenges faced by patients during the posthospital transition, ${ }^{2}$ and further elucidates the differences between patients' expectations and assessments of their resources and goals, and their actual abilities and priorities on discharge. Despite substantial assistance, including housing, food assistance, and case management, Chan Carusone et al. found that the exigencies of day-to-day existence exceeded the patients' capacities to sustain themselves outside the hospital. This failure implies a question: If the interventions alluded to in this study were not enough, then how much more, and how much better, is needed?

Attention to this question of how to best serve high-need patients continues to increase, ${ }^{3}$ and success in intervening to improve care transitions for this population is limited, ${ }^{4}$ in part because providing more care and more coordination requires more resources. Observing the challenges that remain for patients treated in the highly-resourced setting that is Casey House, the authors propose a previously described theoretical construct, minimally disruptive medicine (MDM),${ }^{5}$ as a framework to guide patients and providers in creating a discharge plan that relies on the patient's capacity to integrate disease self-management into his or her daily circumstances. MDM hinges on the concept of balancing workload and capacity: the burden of managing disease

\footnotetext{
*Address for correspondence and reprint requests: Hilary J. Mosher, MFA, MD, lowa City VA Healthcare System, 601 Highway 6 West, Mailstop 111, lowa City, IA, 52246-2208; Telephone: 319-338-0581, ext 7723; Fax: 319-887-4932; E-mail: hilary.mosher@va.gov
}

Received: October 26, 2016; Accepted: November 3, 2016

2017 Society of Hospital Medicine DOI 10.1002/jhm.2679 with the resources and abilities to do so. On first consideration, this seems an attractive approach to operationalizing patient-centered care by tailoring a discharge plan to a patient's goals and capacities. On closer examination, however, MDM, applied to a single transition episode, raises some important concerns.

As Chan Carusone et al. describe, patients may poorly judge their future resources and capacity when making decisions in the hospital setting. Likewise, physicians and other team members may lack insight, perspective, and detailed knowledge of resources and barriers in the outpatient setting. From their vantage point, they may not see the fragile contingencies of the discharge plan that is reflected in the patients' spoken words. At any moment, a well-meant, seemingly well-crafted discharge plan could fall apart.

Within the walls of the hospital, we tend to perform what might be termed maximally disruptive medicine-the treatments provided are exactly those that can't be delivered in a nonhospital setting. For many patients, these interventions are not curative, but rather stabilizing; ${ }^{6}$ we assuage chronic conditions that had become exacerbated by new illness, disease progression, or conditions outside the hospital. To return the patient to his or her home situation, especially one that is under-resourced, with minimized workload can feel counterproductive and demoralizing at best. What prevents one from worrying that, where capacity can't be improved, planning for MDM is, in essence, planning for minimal care?

Viewed in the broader context of a life course health development framework, ${ }^{7}$ which integrates biological, psychological, cultural, and historical experience to explain the development of health trajectories over an individual's lifetime, a minimally disruptive approach might be viewed as amplifying disparities. The patients contributing to the study by Chan Carusone et al. may have arrived in their respective situations through a life course marked by poverty, violence, inadequate housing, poor nutrition, discrimination, and other disadvantages that may have resulted from accident, malfeasance, or choice. Their limited personal capacity and the ongoing chaos that is reflected in many of their comments requires that discharge planning uses imagination and dialogue, with careful, compassionate listening by providers, and close partnering and decision-making by patient and providers. Approaches to building the capacity for such compassion, as well as structural interventions to provide care that is necessary and just for these most vulnerable patients by considering their experiences and beliefs, ${ }^{8}$ remain to be articulated. 
In a sense, the narrative unfolded by Chan Carusone et al. appropriately emphasizes that care transitions contain both complex problems and "wicked" problems. ${ }^{9}$ While aspects of transitions are complex and can be reasonably addressed with complex solutions, these same complex solutions are inadequate to mitigate the seemingly intractable socioeconomic challenges that drive hospital dependence for many highneed patients. Addressing these likely requires a reexamination of what we expect from hospitals, what systems we are able to design and are willing to support to keep people from returning to them, and what it means that for some people returning is the best, and sometimes only, thing to do.

As we continue to seek new models for healthcare in high-need, high-risk populations, we may do well to focus further longitudinal qualitative study on building a deep understanding of when and how patients achieve success following discharge. What characterizes patients, caregivers, service networks, and communities in healthcare settings with the highest rates of effective transitions? Maintaining equilibrium outside an institutional setting is convoluted, time-consuming, nuanced, and taxing; that those who have not experienced doing so as a patient or caregiver might struggle to help others should not surprise us. The concepts of capacity and workload lend themselves to structuring discovery of the resources that patients, not providers and policy-makers, have found through their lived experience to be most crucial to their enduring well-being. Learning from these experiences may shift the balance by increasing our own capacity to understand what constitutes success.

Disclosures: The views expressed in this article are those of the authors and do not necessarily represent the views of the Department of Veterans Affairs. The authors report no conflicts of interest.

\section{References}

1. Chan Carusone S, O'Leary B, McWatt S, Stewart S, Craig S, Brennan D. The lived experience of the hospital discharge "plan": a longitudinal qualitative study of complex patients. J Hosp Med. 2017;12(1):5-10.

2. Kangovi S, Barg FK, Carter T, et al. Challenges faced by patients with low socioeconomic status during the post-hospital transition. J Gen Intern Med. 2014;29:283-289.

3. Blumenthal D, Chernof B, Fulmer T, Lumpkin J, Selberg J. Caring for high-need, high-cost patients - an urgent priority. N Engl J Med. 2016;375:909-911.

4. Powers BW, Milstein A, Jain SH. Delivery models for high-risk older patients: back to the future? JAMA. 2016;315:23-24.

5. Abu Dabrh AM, Gallacher K, Boehmer KR, Hargraves IG, Mair FS. Minimally disruptive medicine: the evidence and conceptual progress supporting a new era of healthcare. J R Coll Physicians Edinb. 2015;45:114-117.

6. Pannick S, Wachter RM, Vincent C, Sevdalis N. Rethinking medical ward quality. BMJ. 2016;355:i5417.

7. Kressin NR, Chapman SE, Magnani JW. A tale of two patients: patient-centered approaches to adherence as a gateway to reducing disparities. Circulation. 2016;133:2583-2592.

8. Thiel de Bocanegra H, Gany F. Good provider, good patient: changing behaviors to eliminate disparities in healthcare. Am J Manag Care. 2004;10:SP20-28.

9. Churchman CW. Wicked problems. Manage Sci. 1967;14(4):B141-B142. 